\section{Restrictive cardiomyopathy and hypertrophic cardiomyopathy overlap: the importance of the phenotype}

\author{
Juan Pablo Kaski, ${ }^{1,2}$ Elena Biagini, ${ }^{3}$ \\ Massimiliano Lorenzini, ${ }^{3}$ \\ Claudio Rapezzi, ${ }^{3}$ Perry Elliott ${ }^{1,4}$ \\ 'Institute of Cardiovascular Science, \\ University College London, UK; \\ ${ }^{2}$ Inherited Cardiovascular Diseases Unit, \\ Department of Cardiology, Great Ormond \\ Street Hospital, London, UK; \\ ${ }^{3}$ Cardiovascular Department, University \\ of Bologna, Italy; \\ ${ }^{4}$ The Heart Hospital, University College \\ London Hospitals, London, UK
}

\begin{abstract}
Restrictive cardiomyopathy (RCM) is defined on the basis of the haemodynamic finding of restrictive ventricular physiology. However, restrictive ventricular pathophysiology is also a feature of other subtypes of cardiomyopathy, including hypertrophic cardiomyopathy (HCM). Clinically and aetiologically, there is an overlap between RCM and HCM with restrictive physiology. However, the clinical distinction between these two entities can be an important pointer towards the underlying aetiology. This review highlights the importance of the recognition of the clinical phenotype as the first step in the classification of cardiomyopathies.
\end{abstract}

\section{Introduction}

Restrictive cardiomyopathy (RCM), the rarest of the cardiomyopathies, is defined as restrictive ventricular physiology (increased ventricular stiffness resulting in a precipitous rise in ventricular pressures with only a small increase in volume) in the presence of normal or reduced diastolic volumes, normal or reduced systolic volumes and normal ventricular wall thickness. ${ }^{1}$ RCM is often characterized by bilateral atrial dilatation, but this is not an invariable feature of the condition. ${ }^{2}$ Restrictive ventricular pathophysiology also occurs in other subtypes of cardiomyopathy such as hypertrophic cardiomyopathy (HCM) and dilated cardiomyopathy (DCM) and in the current issue of Cardiogenetics, Bahl and colleagues propose that these conditions should be considered part of the same disease spec- trum. ${ }^{3}$ Whilst the authors highlight the clinical and genetic overlap between HCM with restrictive physiology and idiopathic RCM, and correctly point out that the treatment and prognosis in many cases is similar, the suggestion that patients with left ventricular hypertrophy and restrictive physiology should be reclassified as RCM is, in our view, potentially misleading and likely to result in confusion in the clinical setting.

\section{The classification concept for cardiomyopathies}

The most recent European Society of Cardiology (ESC) classification for cardiomyopathies, published in $2008,{ }^{1}$ divides the cardiomyopathies into specific morphological and functional phenotypic subgroups. The overriding aim of this classification scheme is to provide a clinically relevant tool with which to diagnose and manage patients and families with heart muscle disease. In the ESC classification scheme, the first step is the recognition of the clinical phenotype. The next tasks are to determine, if possible, the probability of genetic disease and then proceed in a logical way to a specific diagnosis. The classification takes into account that different genetic and non-genetic disorders can result in identical phenotypes and that, in a family context, mixed phenotypes in which different morphological subtypes can occur.

\section{Definition and etiology of restrictive cardiomyopathy}

Of all the cardiomyopathies, RCM has been the most difficult to categorize, in part due to the myriad causes of restrictive ventricular physiology, but also because, unlike other cardiomyopathies that are defined primarily by morphological criteria, the diagnosis of RCM is based on haemodynamic parameters. Alternative definitions based on the presence of bi-atrial dilatation or poorly defined criteria such as mild hypertrophy have been previously suggested, ${ }^{2}$ but are limited by poor specificity.

The most common causes of RCM are summarized in Table 1. As discussed by Bahl et $a l .{ }^{3}$ between 30 and $60 \%$ of cases of RCM are caused by mutations in cardiac sarcomere

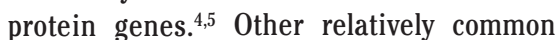
causes include amyloidosis, and, in the tropics, endomyocardial fibrosis. ${ }^{6}$ The prognosis of RCM is generally poor, with a high incidence of congestive cardiac failure, cardiac transplantation and ventricular arrhythmia.,
Correspondence: Juan Pablo Kaski, University College London and Great Ormond Street Hospital, UK. E-mail: j.kaski@ucl.ac.uk

Key words: cardiomyopathy, sarcomere protein disease, classification, diastology.

Received for publication: 3 September 2012. Revision received: 6 September 2012. Accepted for publication: 6 September 2012.

This work is licensed under a Creative Commons Attribution NonCommercial 3.0 License (CC BYNC 3.0).

(C) Copyright J.P. Kaski, et al., 2012

Licensee PAGEPress, Italy

Cardiogenetics 2012; 2:e10

doi:10.4081/cardiogenetics.2012.e10

\section{Should restrictive cardiomyopathy and hypertrophic cardiomyopathy with restrictive physiology be considered separate entities or a single disease?}

The review by Bahl and colleagues ${ }^{3}$ highlights the substantial degree of clinical and genetic overlap between idiopathic RCM and HCM with restrictive ventricular physiology. Clinically, one can observe at least three different types of overlap: i) The same patients can show at a given time a typical morphologic hypertrophic phenotype associated with a restrictive pathophysiology (on echocardiography and/or cardiac catheterization); ii) during the follow up, progression can be observed from a classic HCM to a phenotype characterized by mild residual hypertrophy and restrictive pathophysiology; and iii) within a given family one can observe cases with classic HCM and others with RCM without hypertrophy. However, this should not lead to the conclusion that both conditions are the same and therefore should be classified as one disorder. The distinction between HCM and RCM can be extremely helpful clinically as a pointer towards a specific etiology. For example, the coexistence within the same family of individuals with a RCM phenotype and others with a HCM restrictive phenotype is a red flag to search for sarcomeric disease. While we advocate preservation of the diagnostic label of RCM, we acknowledge that it is sometimes difficult to apply in clinical practice. For example, by restrictive physiology do we mean a specific type of diastolic dysfunction (where myocardial relaxation and the first phase of diastolic filling are normal but that last part of diastole 
Table 1. Common causes of restrictive cardiomyopathy.

\begin{tabular}{ll}
\hline Familial & Non-familial \\
Sarcomere protein mutations & Amyloid (AL/prealbumin) \\
Familial amyloidosis & Scleroderma \\
\hline Desmin mutations & Endomyocardial fibrosis \\
Pseudoxanthomaelasticum & Carcinoid heart disease \\
\hline Fabry disease & Radiation \\
Haemochromatosis & Drugs \\
\hline Glycogen storage disease & Metastatic cancers \\
\hline Adapted from Elliott et al. &
\end{tabular}

is abnormal producing a dramatic increase in filling pressure), or should one include all conditions in which ventricular volumes and ejection fraction are normal, while diastolic filling is impaired? Secondly, conditions such as amyloidosis are often used as a paradigm for RCM, in spite of the fact that amyloidal heart disease is usually characterized by an increase in left ventricular wall thickness and does not, therefore, meet contemporary definitions of RCM. In the past, ambiguous and imprecise concepts such as mild hypertrophy or near-normal systolic function were used to overcome this problem but this resulted in the focus being taken away from the clinical priority of making a diagnosis. Therefore, in the recent ESC position statement, the concept of RCM as a separate disorder is retained, whilst avoiding unnecessary debate on these semantic points. Thus, while merging the entities of RCM and HCM with restrictive physiology, as suggested by Bahl and colleagues, might seem logical, the consequence would be a return to a situation in which we have to resort to terms such as non-hypertrophic hypertrophic cardiomyopathy to describe individual phenotypes.

\section{Conclusions}

As demonstrated by the article by Bahl and colleagues in the current issue of Cardiogenetics, ${ }^{3}$ no single classification scheme for cardiomyopathies is perfect. However, the approach of the ESC position statement, based on the recognition of the clinical phenotype, is relevant to everyday clinical practice. Using this approach, the recognition of the basic clinical phenotype is the first step in the diagnostic pathway and so, whilst acknowledging that there is substantial clinical and genetic overlap between idiopathic RCM and HCM with restrictive ventricular physiology, the underlying principle of recognizing the clinical phenotype remains the key to the classification of heart muscle disease.

\section{References}

1. Elliott P, Andersson B, Arbustini E, et al. Classification of the cardiomyopathies: a position statement from the european society of cardiology working group on myocardial and pericardial diseases. Eur Heart J 2008;29:270-6.

2. Ammash NM, Seward JB, Bailey KR, et al. Clinical profile and outcome of idiopathic restrictive cardiomyopathy. Circulation 2000;101:2490-6.

3. Bahl A, Saikia UN, Khullar M. Idiopathic restrictive cardiomyopathy - perspectives from genetic studies. Is it time to redefine these disorders? Cardiogenetics 2012;2:e4.

4. Kaski JP, Syrris P, Burch M, et al. Idiopathic restrictive cardiomyopathy in children is caused by mutations in cardiac sarcomere protein genes. Heart 2008;94: 1478-84.

5. Mogensen J, Kubo T, Duque M, et al. Idiopathic restrictive cardiomyopathy is part of the clinical expression of cardiac troponin I mutations. J Clin Invest 2003; 111:209-16.

6. Kushwaha SS, Fallon JT, Fuster V. Restrictive cardiomyopathy. N Engl J Med 1997; 336:267-76.

7. Kubo T, Gimeno JR, Bahl A, et al. Prevalence, clinical significance, and genetic basis of hypertrophic cardiomyopathy with restrictive phenotype. J Am Coll Cardiol 2007;49:2419-26. 